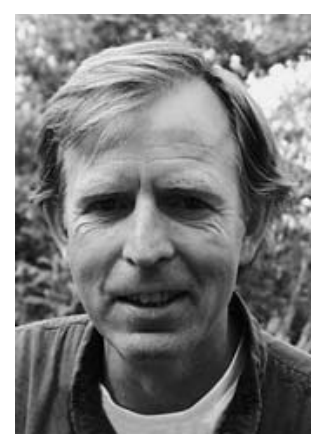

\title{
Behavioural Problems in Children
}

In the last decades behavioural problems in children have soared increasingly into the limelight. New diagnostic terms and categories have been introduced to either be replaced by yet newer ones or be further diversified into different terms and categories.

To a certain extent children labelled as being abnormal show the kind of behaviour adults have always complained about. They can't sit still when they need to learn things they're not interested in; they don't control their impulses as adults do; they talk when you don't want them to or don't talk when you do want them to; they lie about stealing sweets, etcetera. In many cases I was more concerned about those delicate children who never misbehaved than about those raw diamonds who simply showed society was not really attending to their needs and was instead forcing them into a straitjacket.

But, beyond what has probably belonged to all ages and cultures, the increase in serious psychological and psychiatric conditions in children is reason for great concern. This should be seen as the signs and symptoms of a disturbed collective life force. Beyond the individual family histories that may also explain their problems, these children express a dissonance in society at large. Some experts rightfully call the increase in children's disorders like autism and ADHD epidemic.

As every homeopath knows from practice and as several articles in this issue testify, homeopathy is a wonderful system for treating the diversity of symptoms gathered under the common denominator "behavioural problems". However, as long as the causative factors underlying this "behavioural epidemic" remain unidentified and unaddressed within society, all homeopaths can do is to treat the symptoms. Even if in the homeopathic treatment of the individual these were to be fully addressed, resulting in a real cure of the individual child, the collective basically remains as deranged as it was before. The number of children treated with homeopathy is simply too small to make a clear difference in the totality of mankind. Besides that, even if many more children were to receive homeopathic treatment, treating the effects of the cause is still something different from addressing the cause itself.

So long as global healthcare remains based on the allopathic paradigm of suppressing diseases and symptoms through chemical drugs homeopaths can but minimise the damage and hope for change.

To bring about this ultimately unavoidable change there is a major complicating factor, namely the healthcare economy. By largely leaving research and development of healthcare to the free market, health has become subordinate to the stock market. The financial interests involved in letting mankind consume as many drugs and vaccinations as possible have become the determining course-setting force within the health profession. Big Pharma is the result of the influential marriage of capitalism and allopathy, a "high maintenance" union representing a financial power that overextends the budget of many countries, and has enormous authority.

If we look at the percentage of patients using Complementary and Alternative Medicine and compare that with the percentage of funds used for research and development of CAM there is a huge imbalance. The reality of healthcare today is that it is a stock-market-ruled, totalitarian system, in which the allopathic dogma dictates legislation, controls all the funds and successfully marginalises other players in the field.

In this model the patient plays the role of consumer, and the millions of children receiving chemical drugs to change their behaviour are but one sad and cynical example of this. To promote consumption and influence legislation Big Pharma donates millions every year to patient organisations like the US National Alliance on Mental Illness (The New York Times - October 21, 2009). If we look at it more closely it is not the behaviour of children that is the real problem but that of their parents who together uphold a society that itself is autistic, obsessively preoccupied with material gain but with an attention deficit to the real needs of its children.

It will not be the health industry itself that will bring about the change dearly needed. A consumers' revolt will be required and luckily the first signs of that are becoming visible. I have no doubt that many healthcare professionals would love to step out of the current paradigm could they see the possibility and trust the alternative. It is up to homeopathy to present consistent results in the treatment of children with behavioural problems and to prove that homeoprophylaxis is a safe and effective alternative to mass vaccination.

I salute the authors who share their vision and experience in this issue for their contribution to healing mental health care.

Harry van der Zee, Editor 